\title{
Oral Aminolevulinic Acid Hydrochloride
}

National Cancer Institute

\section{Source}

National Cancer Institute. Oral Aminolevulinic Acid Hydrochloride. NCI Thesaurus. Code C99763.

A powder for an oral solution comprised of the hydrochloride salt of 5-aminolevulinic acid (ALA) with a potential application for photodynamic therapy. After oral administration, ALA is converted intracellularly into the photosensitizer protoporphyrin IX (PpIX). Upon exposure to light of appropriate waveleng th (violet to blue range), excited PpIX emits a characteristic red fluorescence which could facilitate guided resection, and generates excited sing let oxygen molecules that could kill cells when appropriate laser dosage is applied. ALA is preferentially taken up by and accumulates in many types of cancer cells compared to normal, healthy cells. Consequently, cancer cells can be visualized and can be distinguished from normal, healthy cells. 